\title{
Efficiency of the Incomplete Enumeration Algorithm for Monte-Carlo Simulation of Linear and Branched Polymers
}

\author{
Sumedha $^{1}$ and Deepak Dhar ${ }^{1}$
}

Received August 29, 2004; accepted March 29, 2005

\begin{abstract}
We study the efficiency of the incomplete enumeration algorithm for linear and branched polymers. There is a qualitative difference in the efficiency in these two cases. The average time to generate an independent sample of configuration of polymer with $n$ monomers varies as $n^{2}$ for linear polymers for large $n$, but as $\exp \left(c n^{\alpha}\right)$ for branched (undirected and directed) polymers, where $0<\alpha<$ 1. On the binary tree, our numerical studies for $n$ of order $10^{4}$ gives $\alpha=0.333 \pm$ 0.005 . We argue that $\alpha=1 / 3$ exactly in this case.
\end{abstract}

KEY WORDS: Self-avoiding walks; lattice animals; Monte-Carlo methods for polymers; percolation on trees.

\section{O. INTRODUCTION}

Monte-Carlo(MC) simulations are a very important tool for studying polymers, as exact results are hard to come by, and are available only for the simplest models. Broadly speaking, MC algorithms fall in two classes: ${ }^{(1)}$ the Metropolis type and the genetic type. The Metropolis type algorithms generate a time sequence of configurations of the polymer using a Markovian evolution. The transition probabilities from one configuration to the next are so chosen that the time average of properties of the system are equal to that from the desired distribution. These may use local moves as in Rouse dynamics, ${ }^{(2)}$ bi-local moves as in the reptation algorithm ${ }^{(3)}$ or nonlocal moves as in the pivot ${ }^{(4)}$ and cut-and-paste ${ }^{(5)}$ algorithms. There is inevitably some correlation between different configurations generated in an evolution. These algorithms become inefficient if

\footnotetext{
${ }^{1}$ Department of Theoretical Physics, Tata Institute of Fundamental Research, Homi Bhabha Road, Mumbai 400005, India; e-mail:\{sumedha,ddhar\}@theory.tifr.res.in
} 
the correlation time becomes very large, e.g. when simulating polymers in a random medium.

In the genetic algorithms, one randomly generates a small random number of configurations in each run. The probability that a given configuration is obtained in a run is proportional to the desired distribution. One repeats the process for many runs to get a large sample. Examples of this type are the enrichment ${ }^{(6)}$ and the pruned-enriched Rosenbluth method(PERM)-like ${ }^{(7)}$ algorithms.

While there have been many studies of linear polymers using various MC techniques like pivot, ${ }^{(4,8,9)}$ PERM, ${ }^{(7)}$ Berreti-Sokal algorithms, ${ }^{(10)}$ branched polymers have been less studied. Algorithms used for simulating linear polymers can often be adapted for branched polymers, but they are usually found to be less efficient. For example, in the pivot algorithm, the acceptance probability of the transformed configuration is found to be much less for branched polymers than for linear polymers. ${ }^{(11)}$ The algorithm does not perform well for branched polymers adsorbed on a surface. ${ }^{(12)}$ Similarly the PERM algorithm seems to work less well for branched polymers than for linear polymers. ${ }^{(13)}$ Incomplete enumeration(IE) is an algorithm belonging to the genetic class of algorithms. It has been used for simulating linear polymers, ${ }^{(14)}$ and for branched polymers. ${ }^{(15,16)}$

A better understanding of the efficiency of MC algorithms for generating branched polymers seems desirable. We will study IE for linear and branched polymers in this paper. We choose the average computer time $T_{n}$ needed to generate one statistically independent sample of desired size $n$ as a reasonable measure of efficiency of the algorithm. The dependence of $T_{n}$ on $n$ is very different for IE for linear and branched polymers. We find that for the linear polymers $T_{n} \sim k n^{2}$, but for branched polymers $T_{n} \sim$ $\exp \left(k n^{\alpha}\right), 0<\alpha<1$. We also discuss an improvement of IE, which we call improved incomplete enumeration (IIE), in this paper. We find that the improvement does not change the asymptotic dependence of $T_{n}$ on $n$ in general. The IIE works better than IE, but the difference is only in the coefficient $k$.

The plan of the paper is as follows. We describe the IE algorithm in Section 1. In Section 2 we discuss the efficiency criterion for MC algorithms in general, and for IE in particular. In Section 3 we study the efficiency of IE analytically for some simple cases where the genealogical tree has a simple recursive structure. We also study IE for self-avoiding walks (SAW) in this section. In all cases we find that $T_{n} \sim n^{2}$. In Section 4 we propose an improved version of the IE algorithm, IIE. For simple random walks $T_{n}=n$ for the IIE algorithm as compared to $T_{n} \sim n^{2}$ for IE. For SAWs, IIE is significantly more efficient and becomes better in higher dimensions, but asymptotic efficiency remains the same and $T_{n} \sim a_{d} n^{2}$ in all dimensions, though the coefficient $a_{d}$ decreases 
with increasing dimension. In Section 5 we study IE for branched polymers or lattice animals on a binary tree. We give heuristic arguments and numerical evidence to show that $T_{n} \sim \exp \left(k n^{1 / 3}\right)$ for large $n$ for branched polymers on a binary tree. We also study IE and IIE numerically for undirected and directed branched polymers on a square lattice in this section. We find that in both cases $T_{n} \sim \exp \left(k n^{\alpha}\right), 0<\alpha<1$. We summarise our results in Section 6.

\section{THE INCOMPLETE ENUMERATION ALGORITHM}

The SAW and lattice animals(LA) are simple lattice models of linear and branched polymers in dilute solutions. In order to study the thermodynamic properties of these polymers, one has to average over all allowed configurations of the polymer of a given number of monomers. The averages are defined with all configurations considered to be equally likely. Since the total number of possible configurations grow exponentially fast with size of the polymer, brute-force exact calculation is possible only for small polymers. The MC methods allows us to study much larger sizes by obtaining a representative sample of the set of configurations and estimate the ensemble averages from the sample average.

The IE algorithm is a simple modification of exact enumeration algorithm for generating polymers. A good exact enumeration algorithm generates all possible configurations exactly once. ${ }^{(17)}$ This is ensured by defining a rule which, given an $n$-site configuration of a polymer, identifies uniquely one of these sites as the 'last added site'. Removing this site must result in an allowed polymer configuration of $(n-1)$ sites. The $(n-1)$ site polymer is called the parent of the $n$-site configuration. We start by imagining that we have arranged all configurations in a genealogical tree, whose nodes are the different configurations of the polymer, such that all polymer configurations of $n$ sites are at level $n$ and are connected to their parent at level $(n-1)$. Clearly, the tree depends on the rule used to define parenthood. For example, Fig. 1 shows a genealogical tree for directed lattice animals on a square lattice for $n \leqslant 4$, using one such choice (see Appendix A for details). In the actual implementation of the algorithm, the whole genealogical tree never needs to be stored in full. Parts of the tree are constructed and erased as we proceed in a depth first search.

As the number of configurations of polymer of size $n$ increases exponentially with $n$, the time required to construct the genealogical tree up to level $n$ in the exact enumeration algorithm increases exponentially with $n$. The basic idea of the IE algorithm is to decrease this time by randomly pruning the genealogical tree.

In IE we choose a set of $(n-1)$ real numbers $p_{i}\left(0<p_{i} \leqslant 1\right)$, for $i=1$ to $(n-1)$. Any bond in the genealogical tree connecting level $r$ to level 


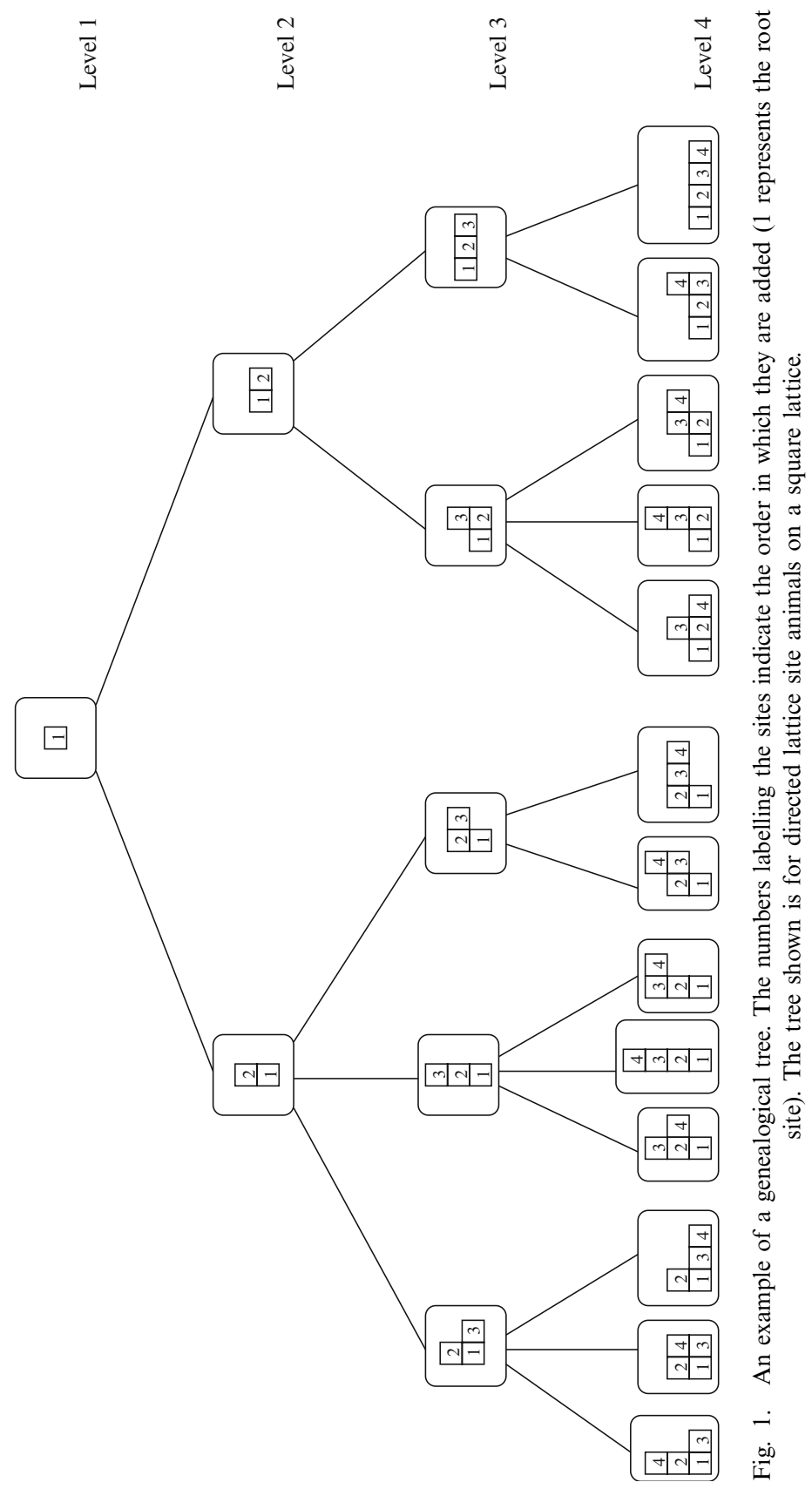


$(r+1)$ is removed with probability $\left(1-p_{r}\right)$ independent of the other bonds. If a configuration gets disconnected from the root node, automatically all its descendants are also removed. We make a depth first search of the pruned genealogical tree up to depth $n$ to determine the different configurations that remain at level $n$. We run the algorithm several times to generate a large sample. The probability of enumeration of a particular $r$ site configuration in a given run is

$$
\Xi_{r}=\prod_{i=1}^{i=r-1} p_{i} .
$$

This is same for all configurations of size $r$. This ensures that the sample of configurations obtained is unbiased. As a configuration can occur at most once in a single MC run, IE samples the population without replacement.

The different runs are mutually uncorrelated. However, the number of configurations produced within one run varies from run to run, and different configurations produced in the same run are correlated. Also, the fraction of runs in which one generates at least one configuration of size $n$ goes down with increasing $n$.

In case of SAWs which model linear polymers, there is a natural labelling scheme in which one just labels the first point of walk by 1 , the second by 2 and so on. In case of branched polymers there are several different choices of labelling possible corresponding to different possible rules of removing a site from a $n$-site cluster to generate a $(n-1)$-site connected cluster. We have used the Martin's labelling scheme ${ }^{(17)}$ for our cluster counting algorithms. A brief description of this can be found in Appendix A.

\section{EFFICIENCY}

In general, in MC methods, the time needed to estimate an ensemble average $\mu=\langle O\rangle$ of some observable $O$ over all clusters of size $n$ averaged over $N$ independent samples would give estimate as $\mu^{*}=\mu \pm \sigma / \sqrt{N}$, where $\sigma^{2}$ is the variance of $O$. If correlations are present, the average time required to estimate $\mu$ within the fractional error $\epsilon$ varies as $(\sigma / \epsilon \mu)^{2} \tau$, where $\tau$ is a measure of correlations in the data. For Metropolis evolution, $\tau$ is the auto-correlation time of the observable $O$. In the case of IE, the efficiency depends on the average time taken by the MC algorithm to generate a single run and the degree of correlations present in the different samples produced in the same run. 
It is difficult to determine the latter exactly for IE. It depends also on the quantity we want to average. Consider a set of configurations generated by $N$ independent runs of the IE algorithm. Let the probability that a single run produces at least one sample be $P(n)$, and the average number of configurations produced per run be $a$. Then for large $N$, we will generate approximately $\mathrm{Na}$ configurations, which will be made of approximately $P(n) N$ mutually uncorrelated groups. Thus the average size of a correlated group is $a / P(n)$. It seems reasonable to measure the efficiency of the algorithm in terms of the average CPU time required to produce one independent group of configurations. This overestimates correlations as this treats all samples produced within one run as fully correlated. $^{2}$

Other definitions of efficiency are possible, and may be advantageous in specific contexts. For example, one may be interested in some asymptotic properties of the polymer problem, like the average branching number $\lambda$, or the critical exponent $\theta$. In this case, the value of $n$ is not decided beforehand, and the desired estimate is obtained by suitable extrapolation of data for different $n$. We can study average number of descendants $\left\langle X_{n}\right\rangle \approx \lambda(1-\theta / n)$ to estimate $\lambda$ and $\theta$. Analysis of errors in such quantities is more complicated, and will not be discussed here.

Let $T_{n}$ be the average CPU time required to obtain one run which generates at least one configuration of size $n$. If $\tau_{n}$ is the average CPU time for one MC run, then we have

$$
T_{n}=\frac{\tau_{n}}{P(n)}
$$

The average CPU time required for one run is estimated easily in terms of the time taken to add or delete a configuration on the genealogical tree. We define this to be one unit of CPU time.

The total CPU time for one MC run is proportional to the number of nodes in the pruned genealogical tree. Let $X_{j}$ denote the random number of $j$-site configurations generated in a single run. The time to visit sites of the randomly pruned tree up-to depth $n$ is $\sum_{j=1}^{n} X_{j}$. The CPU time in a run is then proportional to the number of nodes in the pruned tree. The

\footnotetext{
${ }^{2}$ The value of mean radius of gyration of animals of size 50 on the square lattice is 54.9 and standard deviation $\sigma$ is 26.9. The average number of samples produced per successful run was 27.5. If we calculate the standard deviation of average radius of gyration of $10^{4}$ consecutive runs, we get $\sigma^{\prime}=2.8$. This would have been $\sigma / 100 \approx 0.3$ if they were uncorrelated and 4.6 if they were fully correlated. Thus, assuming fully correlated configurations within a run is not an unreasonable estimate.
} 
average CPU time per run $\tau_{n}$, would be equal to the sum of average values $\left\langle X_{j}\right\rangle$, averaged over all runs.

$$
\tau_{n} \propto \sum_{j=1}^{n}\left\langle X_{j}\right\rangle .
$$

For linear and branched polymers, the total number of configurations $A_{n}$ of a given size $n$ is known to vary as

$$
A_{n} \sim A \lambda^{n} n^{-\theta}
$$

for large $n$. Here $A$ is a constant, $\lambda$ is called the growth constant and $\theta$ is a critical exponent. Since each configuration with $n$ sites has a probability $\Xi_{n}$ (Eq. (1)) of being generated, and there are $A_{n}$ total number of configurations, $\left\langle X_{n}\right\rangle=\Xi_{n} A_{n}$, giving

$$
\tau_{n}=\sum_{j=1}^{n} A_{j} \Xi_{j} .
$$

Since $\left\langle X_{n}\right\rangle$ can be directly estimated in IE, we get a way to estimate the number of configurations $\left\langle X_{n}\right\rangle$ by simulations. This can be used to estimate the $\lambda$ and $\theta$.

A study of the efficiency of the algorithm is complicated as $P(n)$ depends on the structure of the genealogical tree, and is difficult to determine theoretically.

An upper bound on working of these algorithm is the time for exact enumeration of all the samples, which is exponential in $n$. Consider the case in which $p_{i}=p$ for all $i$. So long as $p \lambda>1,\left\langle X_{n}\right\rangle$ will grow exponentially with $n$. As $P(n) \leqslant 1$, this implies that $T_{n}$ increases exponentially with $n$ if $p \lambda>1$. Also, if $p \lambda<1$, then $P(n)$ varies as $(p \lambda)^{n}$ to leading order, but $\tau_{n}$ remains finite $\left(\tau_{n} \geqslant \tau_{1}\right)$. ${ }^{(18)}$ Thus again $T_{n}$ increases exponentially with $n$. These two considerations together imply that a good choice of $p$ is that it should be approximately equal to $1 / \lambda$. However, finding the optimal choice of $\left\{p_{i}\right\}$ for a given problem is non trivial. We investigate this in Section 3 for some illustrative cases.

\section{OPTIMISING THE IE ALGORITHM}

\subsection{Systems with Uniform Genealogical Tree}

The simplest of enumeration problems is the enumeration on a uniform genealogical tree. For example, random walks which are models for linear 
polymers without self-exclusion correspond to a uniform genealogical tree of branching number $\lambda$. The number of nodes at level $n$ is $\lambda^{n-1}$.

Consider a uniform genealogical tree with two descendants per node. In this case number of nodes at level $n$ would be $2^{n-1}$. For the choice of $\left\{p_{i}\right\}$, the probability of connection of root with level $r$, denoted by $P(r)$ follows a simple recursion relation

$$
P(r+1)=2 p_{r} P(r)-p_{r}^{2} P^{2}(r)
$$

with $P(1)=1$. The average $\mathrm{CPU}$ time per run $\tau_{n}$ is given by

$$
\tau_{n}=1+\sum_{i=2}^{n} 2^{i-1} \Xi_{i} .
$$

First we try to find out as to what choice of $p_{i}^{\prime} s$ minimises $T_{n}$ for small $n$.

For small sizes one can try systematic optimisation. Let us choose $n=2$. Then on the binary tree, $P(2)=2 p_{1}-p_{1}^{2}$ and $\tau_{2}=1+2 p_{1}$. This gives

$$
T_{2}=\frac{2 p_{1}+1}{2 p_{1}-p_{1}^{2}}
$$

Minimising with respect to $p_{1}$, we get the minimum value of $T_{2}$ to be $(3+\sqrt{5}) / 2 \approx 2.618$ for $p_{1}=(\sqrt{5}-1) / 2 \approx 0.618$.

Similarly, the time $\left(T_{3}\right)$ of IE for reaching level 3 from level 1, is given by

$$
T_{3}=\frac{1+2 p_{1}+4 p_{1} p_{2}}{2 p_{1}\left(2 p_{2}-p_{2}^{2}\right)-p_{1}^{2}\left(2 p_{2}-p_{2}^{2}\right)^{2}} .
$$

It is easy to check that $T_{3}$ in this case takes its minimum value for $p_{1}=0.534$ and $p_{2}=0.618$. Similarly for $n=4$, the minimum occurs at $p_{1}=0.516, p_{2}=0.534$ and $p_{3}=0.618$. For large $n$, the best choice of $p_{i}$ tends to $1 / 2$. By optimising till $n=30$, we find that the best choice of $p_{i}$ is quite well described by the approximate formula $p_{i} \approx \frac{1}{2}\left(1+0.5 /(n-i)^{2}\right)$.

For large $r$, if $p_{r} \rightarrow p^{*}$, Eq. (6) can be approximated by $P(r+1)=2 p^{*} P(r)-p^{* 2} P(r)^{2}$. For $2 p^{*}<1$, we get $P(r) \rightarrow\left(2 p^{*}\right)^{r}$, and decreases exponentially with $r$. For $\left(2 p^{*}\right)>1$, it leads to $P^{*}(\infty) \sim\left(2 p^{*}-1\right)$.

We have already argued that $p_{i}$ should be close to $1 / \lambda$, else the algorithm is inefficient, $T_{n}$ varies as $\exp (n)$. Consider now the case where $p_{i}=\frac{1}{\lambda}\left(1+\alpha / i^{m}\right)$, where $\alpha$ and $m$ are parameters that we can vary to 
find the optimal values. In this case, $\left\langle X_{n}\right\rangle=\prod_{i}\left(1+\alpha / i^{m}\right)$, and $P(n)$ is approximately given by

$$
\frac{\partial P(n)}{\partial n}=\frac{\alpha}{n^{m}} P(n)-\frac{1}{\lambda^{2}} P^{2}(n)
$$

Then, if $m>1$, we see that $\left\langle X_{n}\right\rangle$ tends to a constant for large $n$, and $\tau_{n}$ is proportional to $n$. Also, $P(n)$ varies as $1 / n$, and we have $T_{n} \sim n^{2}$.

If $m=1$, and $-1<\alpha<1$, then $\left\langle X_{n}\right\rangle$ varies as $n^{\alpha}$, and hence $\tau_{n} \sim n^{\alpha+1}$. Also, Eq. (10) gives $P(n) \sim A(1-\alpha) n^{\alpha-1}$. Interestingly, in the $T_{n}$, these powers cancel and we get $T_{n}=\tau_{n} / P(n) \sim C_{\alpha} n^{2}$. We find that $C_{\alpha} \sim 1 /(1-\alpha)$, hence the best choice of $\alpha$ is $\alpha=0$.

If $m<1$, then $\left\langle X_{n}\right\rangle$ varies as $\exp \left(n^{1-m}\right)$, and $P(n)$ varies as $n^{-m}$, and hence $T_{n}$ varies as $\exp \left(n^{1-m}\right)$ to leading order, thus in this case $m<1$ leads to a suboptimal performance of the algorithm.

On a binary tree for $p_{i}=\frac{1}{2}$, we get $T_{n}=n^{2} / 4$. From systematic optimisation we saw that there exist a nontrivial optimal value for each $p_{i}$ which depends on the depth of the genealogical tree to be reached. This value for uniform binary tree was $p_{i} \approx \frac{1}{2}\left(1+0.5 /(n-i)^{2}\right)$. But even with this choice for large $n$ we get $T_{n} \approx n^{2} / 4$. This result is generalised straight forwardly to $k$-node uniform tree. For the choice, $p_{i}=1 / k \forall i$, we get $T_{n}=\frac{(k-1) n^{2}}{2 k}$.

\subsection{Systems with Recursively Defined Genealogical Tree}

It is necessary to check how nonuniformity of trees can change the above conclusions. The simplest of nonuniform trees are the recursively defined trees. The number of branches from a given node still follow a definite pattern which repeats and depends on the coordination number of the parent node. We consider some examples

A node with $k$ descendants will be called a $k$-node. Consider a tree specified by the rule that the descendants of a 2-node are a 2-node and a 3-node, and the descendants of a 3-node are one 2-node and two 3-nodes. We specify such a tree by the notation $(23,233)$ tree (Fig.1). If $B_{2}(n)$ and $B_{3}(n)$ are respectively the number of nodes at level $n-1$ which have two and three descendants respectively, then

$$
\begin{aligned}
& B_{2}(n)=B_{2}(n-1)+B_{3}(n-1), \\
& B_{3}(n)=B_{2}(n-1)+2 B_{3}(n-1) .
\end{aligned}
$$


From these linear recursion equations it is easy to see that $B_{2}(n)$, $B_{3}(n)$ and also the total number of nodes at depth $n, A_{n}$, all grow as $(\lambda)^{n}$ for large $n$, where $\lambda=(3+\sqrt{5}) / 2)$.

We now look at the efficiency of IE on this tree. Take all $p_{i}=p$. We define $P_{2}(r)$ and $P_{3}(r)$ as the probabilities that a 2-node and a 3-node, respectively, are connected to at least one node $r$ levels below. Clearly they have the following recursions:

$$
\begin{aligned}
& 1-P_{2}(r+1)=\left(1-p P_{2}(r)\right)\left(1-p P_{3}(r)\right), \\
& 1-P_{3}(r+1)=\left(1-p P_{2}(r)\right)\left(1-p P_{3}(r)\right)^{2}
\end{aligned}
$$

with $P_{2}(1)=P_{3}(1)=1$.

For large $r$, near the fixed point we get $P_{2}(r) \approx \frac{p}{1-p} P_{3}(r)$. Substituting in the second equation, we find the linear term vanishes for $p=1 / \lambda$ and the difference equation can be approximated by $\partial P_{2} / \partial r \sim-P_{2}^{2}$, which implies that $P_{2}(n)$ and $P_{3}(n)$ decay as $1 / n$ for large $n$. We get $P_{2}(n) \approx \lambda^{2} /(1+\lambda) n$. The total CPU time at $p=1 / \lambda$ is $(5+\sqrt{5}) n / 10$. It gives the upper bound on time per independent run to be $((\lambda-2)(1+\lambda) /(3 \lambda-2))$ $n^{2} \approx 0.382 n^{2}$.

We can similarly analyse the other recursively defined trees. Consider for example, the tree given by the rule $(23,223)$. We find that growth constant $\lambda$ is 2.4142 and for $p_{i}=1 / \lambda$ for IE this gives $T_{n} \approx 0.396 n^{2}$. On a $(33,233)$ with growth constant 2.732 for $p_{i}=1 / \lambda$ for IE this gives $T_{n}=(\lambda+4) / 4(\lambda+2) n^{2} \approx 0.35 n^{2}$. It is easy to convince oneself that for all recursively defined trees we get $T_{n} \sim n^{2}$.

It is instructive to see the results of systematic optimisation over $\left\{p_{i}\right\}$ in case of nonuniform trees. Similar analysis for $(23,233)$ tree (Fig. 1) between levels 1 and 2 gives $p_{1}=0.618$. Similarly optimising $T_{3}$ between levels 1 and 3 gives $p_{1}=0.562$ and $p_{2}=0.484$. An optimisation between levels 1 and 4 gives the best values of $p_{i}^{\prime} s$ to be $p_{1}=0.562, p_{2}=0.42$ and $p_{3}=0.467$. We see that the optimal value of $p_{i}$ in this case depends on $n$. By optimising till $n=30$, where $n$ is the depth of the genealogical tree, we find that for tree levels away from root and bottom, optimal value of $p_{i}$ approaches $1 / \lambda$ with increasing $i$ and the asymptotic behaviour of algorithm remains the same as long as we choose $p_{i} \approx 1 / \lambda$. The optimal $p_{i}$ values as a function of $i$ are plotted in Fig. 2. The optimising value of $p_{i}$ are a bit higher than $1 / \lambda$ near the two ends $i=1$ and $i=n$. This extra optimisation does not change the $T_{n} \sim K n^{2}$ dependence, and infact does not change the asymptotic value of $K$ either.

The incomplete enumeration algorithm generates a bond percolation process on the genealogical tree, where each link is present independently 


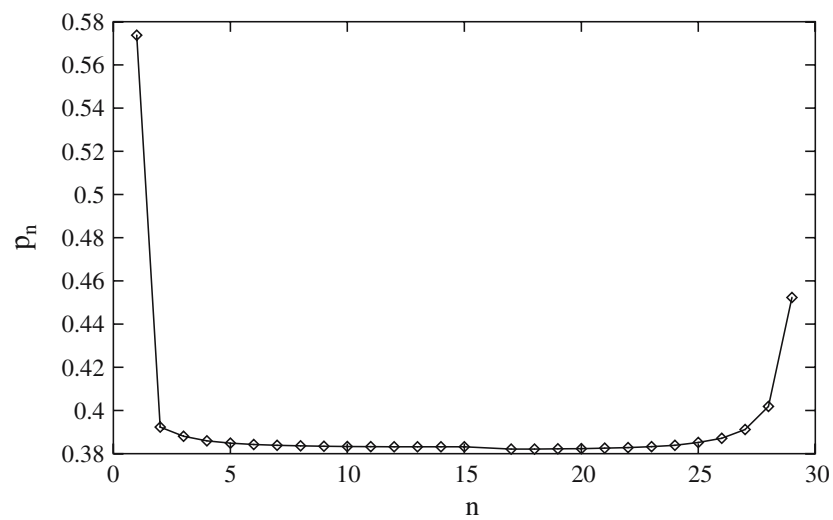

Fig. 2. Plot of optimum values of $p_{i}$ on a $(23,233)$ tree of depth 30.

with a probability $p$. We define the percolation threshold $p_{c}$ on the tree to be such that for all $p>p_{c}$, there is a nonzero probability that the starting node belongs to an infinite cluster. For $p<p_{c}$ the probability of connection between root and level $n$ usually goes down exponentially in $n$. At $p_{c}$ it is expected to decrease as a power law in $n$ and for $p>p_{c}$ it takes a finite value in the limit of $n \rightarrow \infty$. The $p_{c}$ on a tree is bounded from below by $1 / \lambda .^{(19)}$ For the genealogical trees which we discussed, the $p_{c}$ was equal to $1 / \lambda$ and the optimal behaviour of the algorithm was achieved for $p_{i} \approx 1 / \lambda=p_{c}$.

\subsection{Self-Avoiding Walks}

We now consider IE for SAW. For a SAW on a $d$ dimensional lattice, the number of configurations $A_{n} \sim \lambda^{n} n^{\gamma-1}$, where $\lambda$ is a lattice dependent constant and $\gamma$ depends only on the dimension. The exponent $\gamma$ is known to be 1 for $d>4$, and $\gamma=43 / 32$ for $d=2$. ${ }^{(20)}$ The exact value of $\lambda$ is known for the hexagonal lattice, ${ }^{(21)}$ and fairly precise numerical estimate, which matches well with root of a quartic equation with integer coefficients is known on the square lattice. ${ }^{(22)}$

The genealogical tree for SAW is not uniform. For example, for rooted SAW(one end fixed at origin) on a square lattice, the number of different allowed choices of the $n$th step for $n>1$ varies from 0 to 3 , depending on the walk. In this case it is difficult to determine the probabilities of connection up-to level $n$ analytically but we have estimated $P(n)$ numerically by simulations. We choose $p_{i}=\lambda^{-1}(1+1 / i)^{1-\gamma}$, so that on the average we get order one configurations of size $n$ per run for large $n$. With this choice of $p_{i}$ our numerical simulations show that the probability of reaching level $n$ 
goes down as $1 / n$ and hence whenever level $n$ is reached, on an average $\sim n$ SAWs of size $n$ are generated. This also implies that $p_{c}$ is indeed $1 / \lambda$ on the SAW genealogical tree. We did $10^{6} \mathrm{MC}$ simulations and generated walks up-to size 10,000 on a square lattice. We have plotted $T_{n}$ in Fig. 3. Our numerical fit suggests $T_{n}$ for IE to be $(0.42 \pm 0.01) n^{2}$.

In 3-dimensions $\lambda=4.6839$ and $\gamma=1.16^{(20)}$ and nearly $90 \%$ nodes have coordination number 5 . Hence the tree is more uniform than the $2 d$ case and we get $T_{n} \approx 0.43 n^{2}$ (Fig. 3).

The genealogical tree becomes more and more uniform as we go to higher dimensions. In general on a $d$-dimensional hyper-cubic lattice the maximum branching possible is $2 d-1$ and in the limit $d \rightarrow \infty$ the growth constant has an expansion ${ }^{(20)}$

$$
\lambda=2 d-1-\frac{1}{2 d}-\frac{3}{(2 d)^{2}}-\cdots
$$

Hence the dominant branching is $2 d-1$ and probability of a node branching into $2 d-1$ branches increases with dimension, and the lower branching numbers occur with much smaller frequencies. The probability of connection to level $n$ is hard to obtain analytically for any $d$.

In Fig. 3 we have also shown a plot of efficiency of IE in three and four dimensions for SAW. In few hours one can simulate $10^{5} \mathrm{MC}$ runs for walks of size 1000 on a Pentium-4 machine. We get $T_{n} \sim n^{2}$ for two, three and four dimensions. This leads us to conclude that the small nonuniformity of the genealogical tree is unimportant and $T_{n}$ varies as $n^{2}$ in all dimensions for SAW.

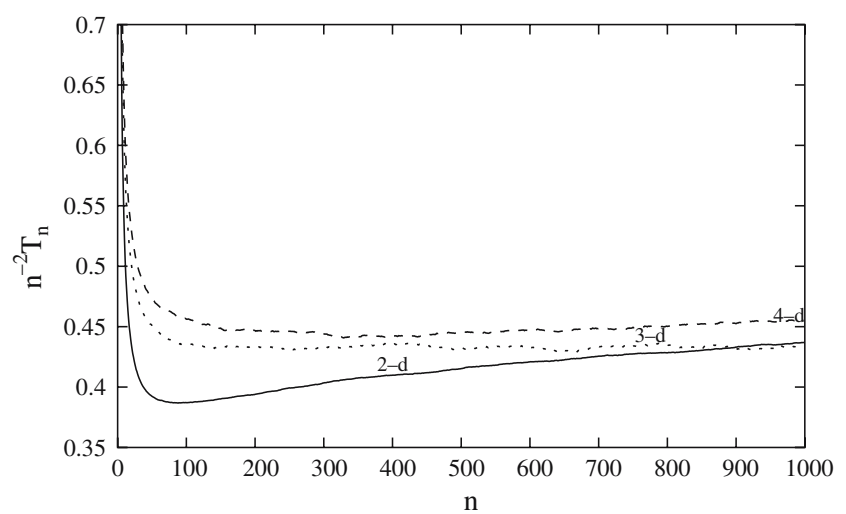

Fig. 3. $T_{n} / n^{2}$ of IE as a function of size $n$ for SAW on a 2-, 3- and 4-dimensional hyper cubic lattice. The lower most graph is for SAW on a square lattice and middle one in $3-d$ and the topmost is for $4-d$. 
We note that for SAWs, other algorithms like pivot are known to be more efficient. For pivot algorithm the correlation time for end to end length varies as $n^{x}$ with $x<1$ in two dimensions. ${ }^{(9)}$ However, if we want to study some variable like correlations in the directions of consecutive steps of the walk, the correlation time will have to satisfy the inequality, $T_{n} \geqslant n$, as one would need to update each step about $O(1)$ times to affect the nearest neighbour correlations.

\section{IMPROVED INCOMPLETE ENUMERATION (IIE)}

The main limitation of IE is attrition: the probability of generating $n$-site configurations in a given MC run goes down with $n$. One way to increase the probability of survival is to redistribute weight amongst the descendants in such a way that while the probability that a particular node is selected remains same as before, the probability that at least one of the descendants is chosen is increased. We call this 'IIE'.

Suppose in the implementation of IE as outlined in Section 1, we come to a node with degree $j$. Then in IE, each link is independently deleted with a probability $(1-p)$, and the probability that all links are deleted is $(1-p)^{j}$, which is nonzero, even if the expected number of descendants of this node is $p j>1$. In IIE, the links are not deleted independently. The probability that any given node is selected remain $p$, but the probability that at least one node is selected increases. This is implemented as follow: if there are $j$ descendants of a node and each link downward is present with probability $p$, then we choose $\operatorname{Int}(p j)$ edges at random and give them weight one, and select one of the edge out of the remaining $j$ at random and give it a weight one with probability $\operatorname{frac}(p j)$ and delete all the other edges.

Hence we see that in IIE, though the average probability of selection of an edge remains $p$, but it enhances the probability of connection between two level of the genealogical tree and hence the probability of success in a given MC run. For example, as will be discussed in Section 4.1 , on a regular tree with $p=1 / \lambda$, the probability of connection up-to $n$ levels below in IIE is exactly one whereas it goes as $1 / n$ in IE.

\subsection{Systems with Recursively Defined Genealogical Tree}

In IIE one redistributes the sum of probabilities of connection from a node to the next level. On a uniform binary tree $y_{i}=2 \forall i$ and with $p_{i}=1 / 2$, $y_{i} p_{i}=1$ and hence for $p_{i}=1 / 2$ with IIE probability of reaching any level $n$ of the tree after $n$ steps is exactly one and exactly one configuration of any given size is generated in the process and hence $T_{n}=n$. With $p_{i}=1 / k$ this result holds for any $k$ node uniform tree. Clearly $p_{i}=1 / k$ is the best choice in this case, as an absolute lower bound on time $T_{n}$ of the algorithm is $n$. 
If we use the improved algorithm for a $(23,233)$ tree, $\left\langle X_{n}\right\rangle$ and hence the average CPU time per run will remain the same. We can also determine the connection probabilities $P_{2}(n)$ and $P_{3}(n)$. The coupled difference equations for $P_{2}(r)$ and $P_{3}(r)$ have no cubic term. The recursions are

$$
\begin{aligned}
& P_{2}(r+1)=p\left(P_{2}(r)+P_{3}(r)\right), \\
& P_{3}(r+1)=p\left(P_{2}(r)+2 P_{3}(r)\right)-\frac{3 p-1}{3}\left(2 P_{2}(r) P_{3}(r)+P_{3}^{2}(r)\right),
\end{aligned}
$$

which at $p=1 / \lambda=p_{c}$ gives $P_{2}(n)$ varying as $1 / n$ for large $n$. The time per independent run comes out to be $(\lambda(3-\lambda) / 3) \approx(1 / 3)$ times that in incomplete enumeration. That is, IIE is nearly three times more efficient than IE.

The IIE certainly works better than IE. But, except for the uniform tree, the difference between IE and IIE is only in the coefficient of $n^{2}$. While performance of IIE improves as the genealogical tree becomes more and more uniform, there is no qualitative difference in the efficiency of IE and IIE on a recursively defined nonuniform tree.

\subsection{IIE for SAW}

We studied IIE on a $d$ dimensional hyper-cubic lattice for $d=2-10$.

IIE enhances the performance of the algorithm by increasing the probability of connection between root and level $n$. For SAW on a square lattice, Fig. 4 shows the probability of connection $P(n)$ for IE and IIE both. $P(n)$ is roughly 3.5 times bigger for IIE. In two dimensions, $T_{n}$ is of order $0.12 n^{2}$ for IIE. In three dimensions the performance is even better and $T_{n} \approx 0.056 n^{2}$, which is roughly a factor of 7.5 less than the time taken by IE.

In general we find on a $d$-dimensional hypercube IIE has a efficiency $T_{n}=a_{d} n^{2}$ where $a_{d}$ is a decreasing function of dimension for generating SAWs. Figure 5 shows the plot of IIE for dimensions 2-10. The memory requirement of the algorithm just increases linearly with system size in all dimensions and we could perform $10^{5} \mathrm{MC}$ runs for walks up-to sizes 1000 in few hours of computer time on a Pentium-4 machine. We find that $a_{d}$ decreases as $d^{-2}$ approximately, i.e. the algorithm performs better with increasing dimension.

We conclude that for IE and IIE for SAW, $T_{n}=a_{d} n^{2}$. The probability of connection between root and level $n$ does not depend on $\gamma$. It depends only on the nonuniformity of the tree. The genealogical tree is more uniform in higher dimensions and the constant $a_{d}$ depends on dimension. For IE, the change in $a_{d}$ with dimension is quite insignificant. But $a_{d}$ can be decreased significantly by redistributing weights. This is a strong 


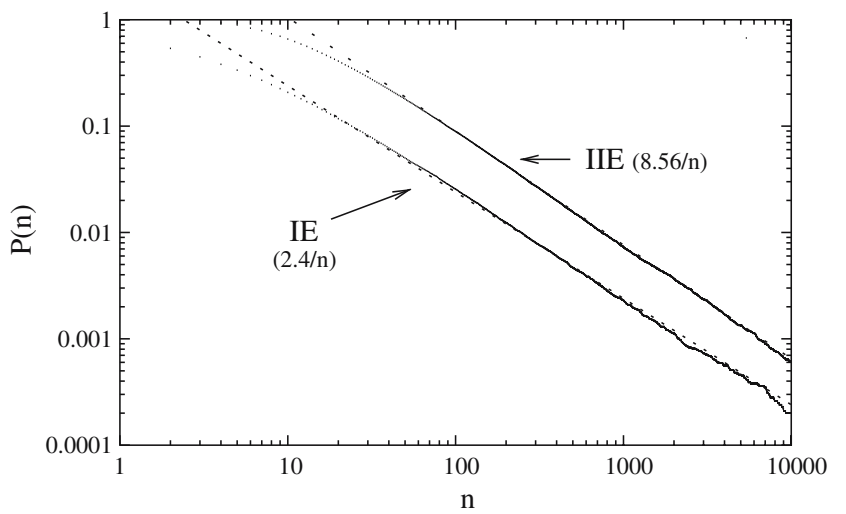

Fig. 4. Probability of getting a walk of size $n$ on a square lattice for IE and IIE.

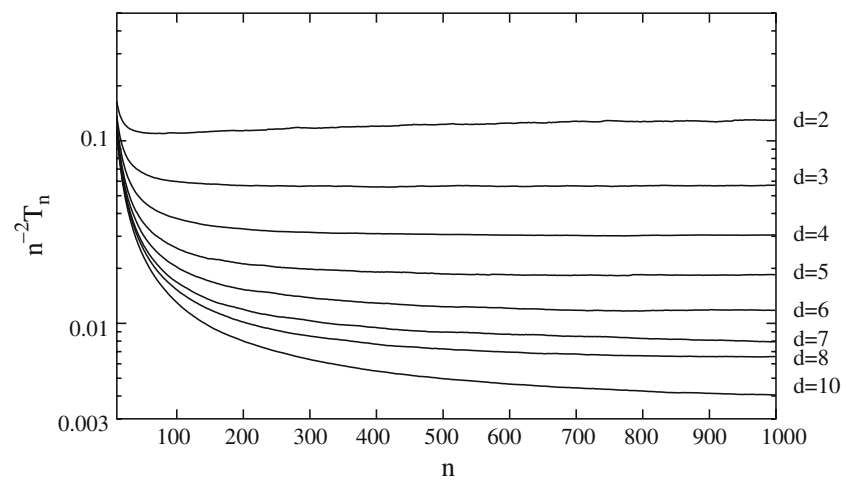

Fig. 5. $T_{n} / n^{2}$ of IIE versus size $n$ for SAW on a 2-8- and 10-dimensional hyper cubic lattice.

numerical evidence that the performance is always $O\left(n^{2}\right)$ independent of the dimension and $\gamma$ for linear polymers.

A further enhancement can be achieved by choosing the pruning only after looking deeper, but we found that because of the increase both in the memory requirement and in the CPU time to generate one configuration, there is no net gain over IIE.

\section{LATTICE ANIMALS AND BRANCHED POLYMERS}

In this section we will study the IE algorithm for branched polymers. Since the efficiency of IE is polynomial in $n$ for linear polymers, it seems plausible that it will be so also for branched polymers. There are two 
important ways in which the genealogical tree for branched polymers differ from that for linear polymers. There are several equally reasonable, computationally easy to implement choices of rules to define parentage, and in all of them the degree of a node is not bounded. The number of possible descendants of a node is of the order of its perimeter sites and hence the maximum of the degree of nodes at level $n$ increases linearly with $n$. The average number of descendants $\lambda$ is of $O(1)$, and the number of nodes with large branching number is exponentially small. But this makes an important difference in the fluctuations of the number of animals of a given size generated in a given run.

The structure of genealogical tree for lattice animals is more complex than for SAWs. We studied the algorithm on genealogical tree obtained by using Martin's labelling scheme. ${ }^{(17)}$ We have tried two or three variations of the priority rules, and our results are insensitive to these changes.

\subsection{Lattice Animals on a Binary Tree}

We first discuss our results for the animals on a binary tree. This simple case is more analytically tractable. The generating function of total number of LA on a binary tree is well known ${ }^{(19)}$ and it is $A(y)=\sum_{0}^{\infty} A_{r} y^{r}=(1-\sqrt{1-4 y}) / 2 y$, where $A_{r}$ is the total number of animals with $r$ sites. The $A_{r}$ are the Catalan numbers, which come up in many other contexts in combinatorics. ${ }^{(23)}$ For large $r$ this gives $A_{r} \sim 4^{r} r^{-\frac{3}{2}}$. The growth constant $\lambda$ in this case is 4 .

The number of descendants of a node at level $r$ in the genealogical tree for this problem lies between 2 to $(r+1)$. In this case the genealogical tree is easily characterised: the root site is a 2 -node. A $k$-node has $k$ descendants, and the degree of these descendants are $k+1, k, \ldots, 3,2$, respectively. This is seen as follows: the node corresponds to a branched polymer with $k$ unblocked perimeter sites, which are ordered by some priority rule. The $m$ th descendant of this node is a node of degree $(k+2-m)$ and corresponds to first $(m-1)$ perimeter sites blocked, $m$ th site occupied and $(k-m)$ allowed for further occupation. Since on a binary tree every site has two downward neighbours, hence we see that a $k$-node will give rise to nodes with $k+1, k, \ldots, 2$ descendants. For example, in Fig. 6, the top node corresponds to an animal of one site, and has two growth sites. If first of these two sites is occupied, then the corresponding animal has three growth sites. If it is blocked it has two growth sites and so on.

The total number of nodes at a level $r$ is equal to $A_{r}$. Let $B_{r}(k)$ is the number of $k$-nodes at level $(r-1)$. We can determine the distribution of the branching number. We find that $B_{r}(k)$ satisfy the following relation 


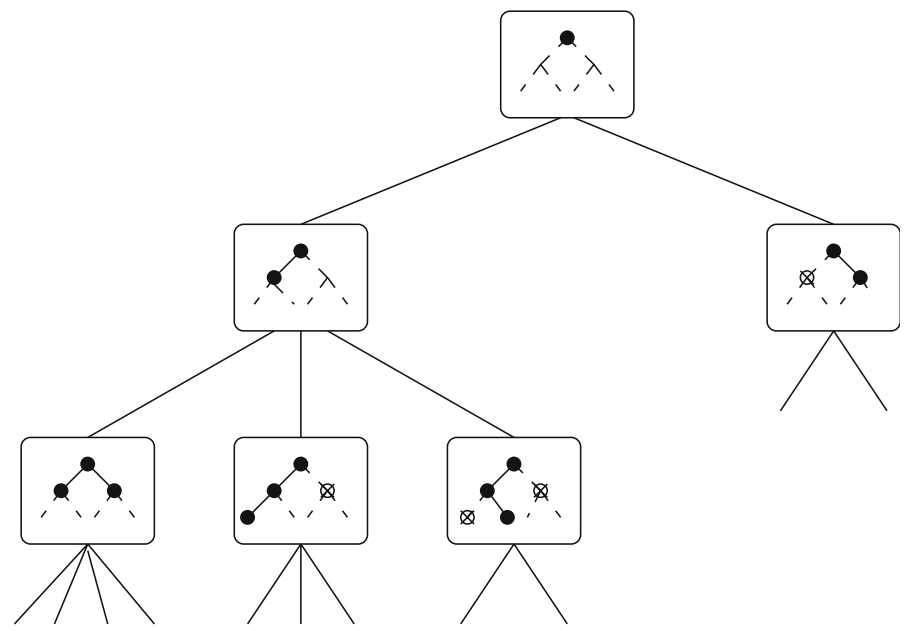

Fig. 6. First few levels of the genealogical tree for lattice animals on a binary tree. Solid circles represent the occupied sites and crossed circles denote blocked sites on the Bethe lattice. Dotted lines sketch the underlying Bethe lattice, whereas solid lines represent the bonds present.

$$
B_{r}(k)=A_{r-2}-\sum_{s=2}^{k-2} B_{r-1}(s)
$$

As $r \rightarrow \infty, 1 / 4$ of the nodes at a level have two offsprings and 1/4 of the total nodes have three offsprings. And level $r$ has exactly one node with degree $(r+1)$. For $k \geqslant 4$, it can be shown that in the asymptotic limit $(r \rightarrow \infty)$, the fraction of nodes having $k$ offsprings is $(k-1) / 2^{k}$ for $r \gg k$.

To find the efficiency factor $T_{n}$, we have to determine the probability of connection of root to a level. If $P(k, r)$ is the probability of a node with $k$ offsprings to be connected to at least one node $r$ levels below it, then $P(k, r)$ has a recursion

$$
P(k, r+1)=1-\prod_{s=2}^{k+1}(1-p P(s, r)), \quad k=2 \text { to } \infty
$$

with initial conditions

$$
P(k, 1)=1 \quad \forall k \geqslant 2
$$

and $p$ is the probability with which we choose any edge of the tree. $P(2, r)$ will give the probability of connection of the root to level $r$ on the genealogical tree. Equation (19) is a nonlinear equation. This equation can also be written as 


$$
1-P(k, r)=(1-P(k-1, r))(1-p P(k+1, r-1)), \quad k>2 .
$$

This equation is also valid for $k=2$ if we choose the convention that $P(1, r)=p P(2, r-1)$.

These equations have the following properties:

1. For $p<1 / 4, P(k, r)$ tends to zero as $r$ tends to infinity exponentially fast for any fixed $k$. In fact, if we consider $r$ as a time like variable and $k$ as space like variable, then $P(k, r)$ has a travelling front solution in this regime $(P(k, r) \cong F(k-v r))$.

2. For $p=1 / 4$, the velocity of travelling front goes to zero. The distance moved by the front increases as $r^{1 / 3}$ and $P(k, r) \sim F\left(k-r^{1 / 3}\right)$. As $F(x) \sim \exp (x)$ for $x \rightarrow-\infty$, this implies that $P(2, r) \sim \exp \left(-c r^{1 / 3}\right)$ for large $x$.

3. For $p>1 / 4$, as $r$ goes to infinity, $P(k)$ tends to a nontrivial fixed point function $P^{*}(k)$ greater than zero.

This may be seen as follows. The fixed point equation in terms of fixed point variables $P^{*}(k)$ is

$$
1-P^{*}(k)=\left(1-P^{*}(k-1)\right)\left(1-p P^{*}(k+1)\right) .
$$

Clearly, $P^{*}(k)=0 \forall k$ is a trivial fixed point of this equation. For $p>1 / 4$, there is a nontrivial fixed point with $P^{*}(k)$ nonzero monotonic increasing, with $P^{*}(k) \approx 1-(1-p)^{k}$ for large $k$. However, a closed form solution for any $p>1 / 4$ is difficult.

On numerically iterating Eq. (19) in $r$, we find that the equation has a travelling front solutions for $p \leqslant 1 / 4$ and has nontrivial fixed point for $p>1 / 4$.

Equation (22) has two stationary solutions, i.e. $P^{*}(k)=1 \forall k$ and $P^{*}(k)=0 \forall k$. For $p \leqslant 1 / 4, P^{*}(k)=0$ is the stable solution while $P^{*}(k)=1$ is an unstable solution. Our initial conditions given by Eq. (20) are steep. Starting with these initial conditions, on numerical iteration we find that as $r$ increases, a front separating stable solution $P=0$ and unstable solution $P=1$ moves in the forward direction. From the translational invariance of Eq. (19) one expects a running wave solution. We find that the front moves with a constant velocity and hence, $P(k, r)$ for large $k$ and $v$ must tend to the asymptotic form

$$
P(k, r) \sim F(k-v r) .
$$




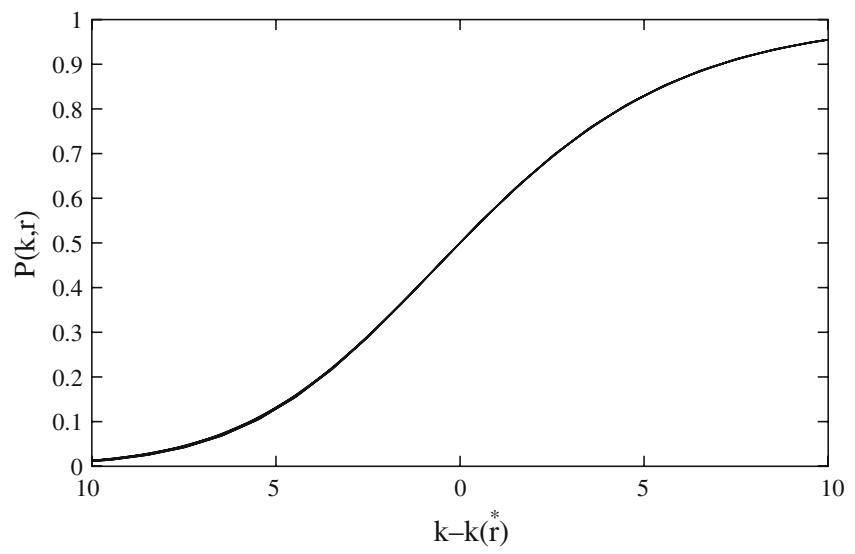

Fig. 7. Plot of $P(k, r)$ versus scaled $k-k^{*}(r)$, for $p=0.25$ and $p=0.25 \pm 0.0001$ and $r=100,300$ and 600 . All the nine curves collapse to the same front profile.

We define $k^{*}(r)$, the width of the front by the equation,

$$
P\left(k^{*}(r), r\right)=\frac{1}{2}
$$

Figure 7 shows a plot of numerically determined $P(k, r)$ with respect to $k-k^{*}(r)$ for $p$ near $1 / 4$. Curves for $p$ below, above and at $p=1 / 4$ all collapse on the same line. Actually, a travelling front for $P(k, r)$ as defined by Eq. (21) exists for all $k,-\infty<k<\infty$, if we take boundary conditions such that $P(-\infty, r)=0$ and $P(\infty, r)=1$.

At $p=1 / 4$, the velocity of the travelling front is zero. If we plot $P(k+1, r)$ as a function of $P(k, r)$, we find that as $r$ increases the graph approaches a limiting form. Thus for the asymptotic wavefront, $P(k+1, r)$ is a single valued nonlinear function of $P(k, r)$. We have plotted these values for different $r$ in Fig. 8 and they all are very close and seem to lie on the same curve. Hence if we start from a point on this curve and iterate the fixed point equation (22) with $p=1 / 4$, we generate a travelling front. We have not been able to deduce the functional form of this function, which corresponds to a first order difference equation for $P^{*}(k)$ from the second-order equation (22). Equation (22) turns out to be a stiff equation and one has to be careful while iterating it in increasing $k$ direction. We iterated Eq. (22) starting with different sets of values of $P^{*}(k+1)$ and $P^{*}(k)$ given by Fig. 8 and found the equation yields a travelling front same as the one shown in Fig. 7. 


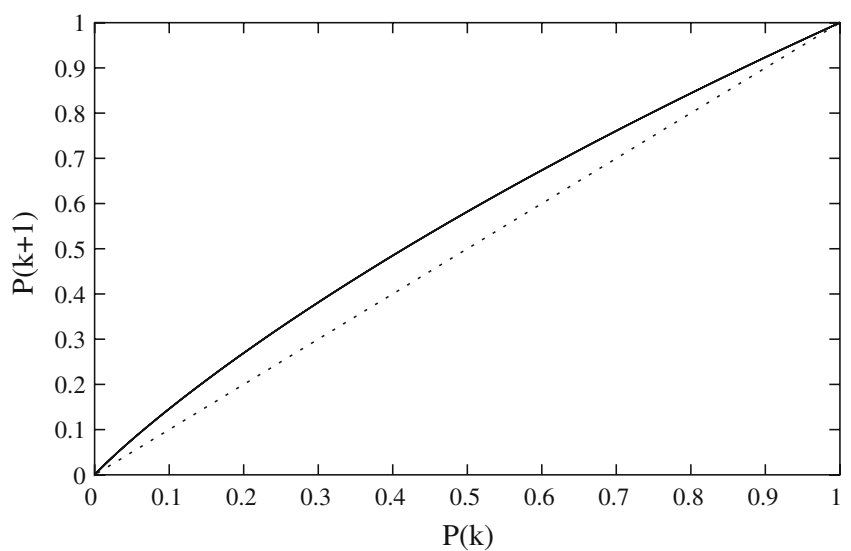

Fig. 8. Plot of $P(k+1)$ as a function of $P(k)$ at $p=1 / 4$ for $r=25000,26000,28000$ and 30000. All the curves are very close and approach a limiting form with increasing $r$. The dotted line is just the line $x=y$.

We could not solve the full nonlinear difference equation (19). Keeping only the terms linear in $P$ will give an upper-bound on $P(k, r+1)$, i.e.

$$
P(k, r+1) \leqslant p \sum_{s=2}^{k+1} P(s, r) .
$$

We can represent this set of equations in matrix form also. Hence if $\mathcal{P}_{r}$ represents the infinite column array with $k$ th entry being $P(k, r)$ then

$$
\mathcal{P}_{r} \leqslant p^{r} M^{r} \mathcal{P}_{0}
$$

where $M$ is the transition matrix. If $\lambda_{m}$ is the largest eigenvalue of $M$ then for $p<1 / \lambda_{m}$, in the limit of $r \rightarrow \infty, \mathcal{P}^{*}$ will be 0 , i.e. $P^{*}(k)=0$ for all $k$, and for $p<1 / \lambda_{m}$.

The elements $M_{i, j}$ of the transition matrix $M$ are such that, $M_{i, j}=1$ for $j \leqslant(i+1)$ and 0 otherwise. If we truncate $M$ beyond $n \times n\left(M_{n}\right)$, then the determinant $D_{n}$ of $M_{n}$ comes out to be

$$
D_{n}=A(\lambda)\left[\frac{1}{x_{1}^{n+1}}-\frac{1}{x_{2}^{n+1}}\right]
$$

with $x_{1}, x_{2}=\frac{-1}{2} \pm \sqrt{1-\frac{4}{\lambda}}$, and $A(\lambda)=1 / \sqrt{1-\frac{4}{\lambda}}$ is a coefficient which does not depend on $n$. Then equating $D_{n}=0$ in the $n \rightarrow \infty$ limit gives $\lambda_{m}=4$. This 
implies that for $p<1 / 4, P(k, r)$ will decay exponentially with increasing $r$ and Eq. (25) will work well. Hence, by definition percolation threshold $p_{c}$ of this tree is $1 / 4$.

The linearised recursion can be solved explicitly, and we get,

$$
P(k, r)=p^{r} \frac{\Gamma[k+2 r-1]}{\Gamma[k+r-1] \Gamma[r+1]}
$$

which for large $r$ gives

$$
P(k, r) \sim \frac{1}{4 \sqrt{\pi r}} \exp \left[\ln 2\left(k+r \frac{\ln (4 p)}{\ln (2)}\right)\right] .
$$

If we assume a travelling front solution of kind $P(k, r) \propto \exp (\lambda(k-v r))$ to be valid in the tail of the distribution, then substituting in linearised recursion (Eq. (25)), for a given $p$ we get a spectrum of travelling wave like solutions parametrised by $\lambda$ with the velocity $v$ of the front given by

$$
v=\frac{1}{\lambda} \ln \frac{1-\exp (-\lambda)}{p}-1 \text {. }
$$

In this case, it is known that the front actually chooses a unique velocity given by minimum of right-hand side of Eq. (30) with respect to $\lambda .{ }^{(24)}$ The front velocity is given by

$$
v^{*}=\frac{2 \exp \left(-\lambda^{*}\right)-1}{1-\exp \left(-\lambda^{*}\right)}
$$

where $\lambda^{*}$ is the solution of the transcendental equation

$$
\frac{-1}{\lambda^{*}} \ln \frac{1-\exp \left(-\lambda^{*}\right)}{p}+\frac{\exp \left(-\lambda^{*}\right)}{1-\exp \left(-\lambda^{*}\right)}=0
$$

Near $p=1 / 4$, we can take $v \approx \ln (4 p) / \ln (2)$ and $\lambda \approx \ln 2$. Travelling front solutions have been found in a large variety of problems in physics. ${ }^{(25)}$

The linearisation of Eq. (19) would be valid only for $p \leqslant 1 / 4$ and $k<k_{o}(r)$. Beyond that, linear solution will grow beyond one whereas the solution of the full nonlinear equation will saturate to 1 . Here $k_{\mathrm{o}}(r)$ is the value of $k$ at which $P(k, r)$ given by Eq. (29) becomes of $O(1)$ and is equal to

$$
k_{\mathrm{o}}(r)=\frac{-r \ln (4 p)}{\ln 2}, \text { for } \quad p<\frac{1}{4}
$$


At $p=1 / 4$, the asymptotic velocity of the front is zero and the front advances as a sub linear power of $r$. This is the critical point of the percolation on this tree, and Eq. (29) gives a algebraic decaying solution for sufficiently small $k$. This is only an upper bound to the actual value. On numerically iterating Eq. (19) for $r$ up to order $10^{4}$, we found unexpectedly that it decays as a stretched exponential in $r$.

The fixed point equation as given by Eq. (22) is again a nonlinear equation. To find the dependence of probability of connection of root, $P(2, r)$, on the width of the front we solved the linearised fixed point equation. On solving, we find that it goes as $2^{-k^{*}(r)}$ for large $r$, where $k^{*}(r)$ is the width of the distribution. Hence in general, $P(2, r) \sim \exp \left(-a k^{*}(r)\right)$.

We further studied the width $k^{*}(r)$ of the front as a function of $r$ for different values of $p$. At $p=1 / 4$ we found $k^{*}(r) \sim r^{1 / 3}$. Figure 9 shows a plot of $k^{*}(r)$ as a function of $r^{1 / 3}$. For $p=1 / 4$, the plot is a straight line. This implies that $P(2, r) \sim \exp \left(-c r^{1 / 3}\right)$ at $p=1 / 4$. For $p<1 / 4, k^{*}(r)$ varies linearly with $r$ and tends to a constant for $p>1 / 4$. We can directly iterate Eq. (19). In Fig. 10 we have plotted $-\log (P(2, r))$ as a function of $r^{1 / 3}$ which comes out to be a straight line. Figures 9 and 10 are strong numerical evidence that the probability of connection goes as $\exp \left(-c r^{\alpha}\right)$ for branched polymers on binary tree. Our numerical studies give $\alpha=0.333 \pm 0.005$ and $c=2.47 \pm 0.01$.

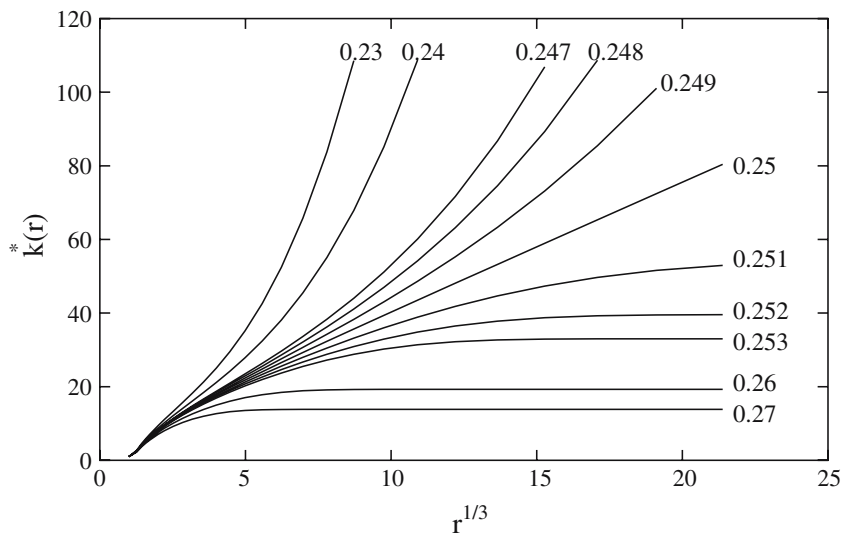

Fig. 9. The width $k^{*}(r)$ of the travelling front as a function of $r^{1 / 3}$ for different values of $p$. The value of $p$ increases from left to right. Curves of left of $p=1 / 4$ are for $p<1 / 4$ and the ones on right are for $p>1 / 4$. For $p=1 / 4$ the graph approaches a straight line as $r \rightarrow \infty$. 


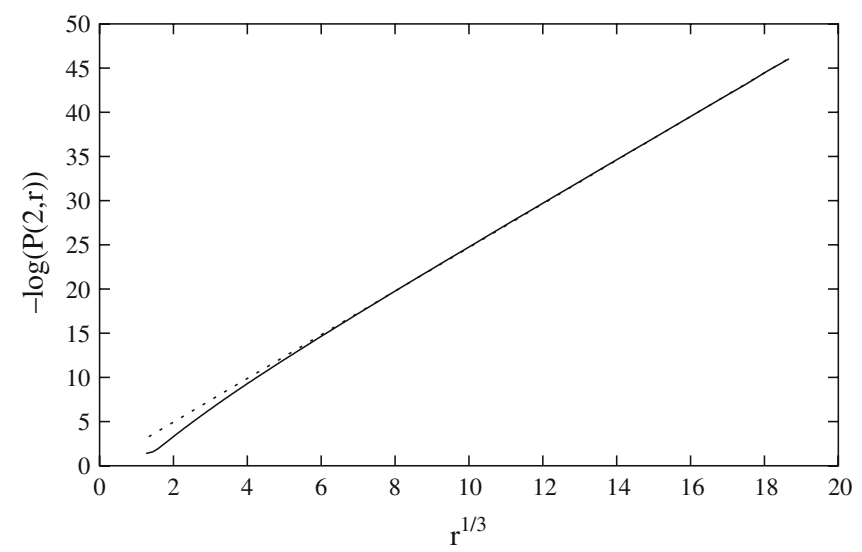

Fig. 10. Thick line is the plot of $-\log (P(2, r))$ as a function of $r^{1 / 3}$, when $p$ is taken to be $1 / 4$. The dotted line is a straight line of slope 2.47 .

\subsection{Heuristic Argument for the Stretched Exponential Behaviour of $P(n)$ at $p=1 / 4$}

We now present a heuristic argument to understand why $k^{*}$ varies as $r^{1 / 3}$ at $p_{c}$. Let us consider a genealogical tree of LA on a binary tree, in which nodes with more than $k$ descendants are deleted. We denote the probability that the maximum degree of a node connected to root down to level $r$ is $k_{m}$, by $H_{2}\left(k_{m}\right)$ and the probability that a $k_{m}$ node is connected to at least one node $r$ level down on the truncated genealogical tree by $J_{k_{m}}(r)$.

Now on a truncated tree, transition matrix $M$ is no longer infinite. It is now a $k_{m} \times k_{m}$ matrix with $M_{i, j}=1$ for $j \leqslant(i+1)$ and 0 otherwise. Here $M_{i, j}$ represents the $i$ th row and $j$ th column entry of $M$, and we find the critical value of $p$ which is just inverse of the largest eigenvalue of $M$ to be a function of $k_{m}$ and is equal to

$$
p_{c}\left(k_{m}\right)=\frac{1}{4}\left(1+\tan ^{2}\left(\frac{\pi}{k_{m}+1}\right)\right) .
$$

For $p<p_{c}\left(k_{m}\right), J_{k_{m}}(r)$ decays exponentially with $r$. In large $r$ limit it is given by

$$
J_{k_{m}}(r) \sim \exp \left(r \log \left(p / p_{c}\left(k_{m}\right)\right)\right)
$$

At $p=1 / 4$, we get $J_{k_{m}}(r) \sim \exp \left(-b r / k_{m}^{2}\right)$, where $b$ is a constant. 
It is easy to get a lower bound on $H_{2}\left(k_{m}\right)$, as a order $k_{m}$ node occurs first time at level $k_{m}$ and probability of connection of root to this node is $p^{k_{m}}$. Hence

$$
H_{2}\left(k_{m}\right) \geqslant p^{k_{m}}=4^{-k_{m}}
$$

Hence, since $p=1 / 4$ is less than $p_{c}\left(k_{m}\right)$ for any finite $k_{m}$, $J_{k_{m}}(r) \sim \exp \left(-b r / k_{m}^{2}\right)$, where $b=\pi^{2}$. Since $H_{2}\left(k_{m}\right) \geqslant \exp \left(-a_{p} k_{m}\right)$, for large $r$ we get

$$
P(2, r) \geqslant \max _{k_{m}}\left[\exp \left(-a_{p} k_{m}-\frac{b r}{k_{m}^{2}}\right)\right]
$$

which gives

$$
P[2, r] \geqslant \exp \left(-c r^{1 / 3}\right)
$$

where $c=\frac{3}{2}\left(2 b a_{p}^{2}\right)^{\frac{1}{3}}$. If we take $H_{2}\left(k_{m}\right)$ to be as given by Eq. (36), we get an lower bound on $P(2, r)$. Taking $b=\pi^{2}$ and $a_{p}=\log 4$ we get $c=\frac{3}{2}\left(2 \pi^{2} \log ^{2} 4\right)^{1 / 3}=5.04$. This should be compared with the numerical estimate $c \cong 2.47$.

Thus our numerical simulations and qualitative arguments show that probability of connection goes down as a stretched exponential at $p=1 / 4$, the $p_{c}$ of the genealogical tree of LA on binary tree as opposed to $r^{-1}$ decay for linear polymers. So if we chose $p_{i}=1 / 4 \forall i$, then $\left\langle X_{r}\right\rangle \sim r^{-3 / 2}$ and hence the average computer time to generate one statistically independent sample of size $r, T_{r}$ would go as $\exp \left(c r^{1 / 3}\right)$ to leading order.

Clearly the algorithm is not working well and one would like to enhance its efficiency if possible. We tried to study the algorithm by choosing $p_{i}$ such that its asymptotic value is $1 / 4$. We chose $p_{i}=\frac{1}{4}\left(1+\frac{x}{i^{m}}\right)$ and studied $T_{r}$ as a function of $x$ and $m$.

As argued earlier, taking $m=1$, we can change $\tau_{r}$ and $P(r)$ by multiplicative factors which are powers of $r$. This will not make much of a difference, as the leading dependence remains $\exp \left(c r^{1 / 3}\right)$. Using $m<1$, seems to be more interesting.

For $m<1$, the average CPU time per MC run would vary as $\exp \left(x r^{1-m}\right)$. In case of linear polymers, we saw that time complexity of the algorithm for $m=1$ for any $x$ is polynomial in $r$. Hence, $m<1$ was clearly a bad choice. But in the case of LA, this increase in numerator is exactly cancelled by a corresponding increase in $P(r)$. For $2 / 3 \leqslant m<1, \tau_{r}$ increases as $\exp \left(x r^{1-m}\right)$ and $P(r)$ varies as $\exp \left(-c r^{1 / 3}+x r^{1-m}\right)$ to leading order for large $r$. These cancel to give $T_{r} \sim \exp \left(c r^{1 / 3}\right)$ independent of $m$. To monitor 


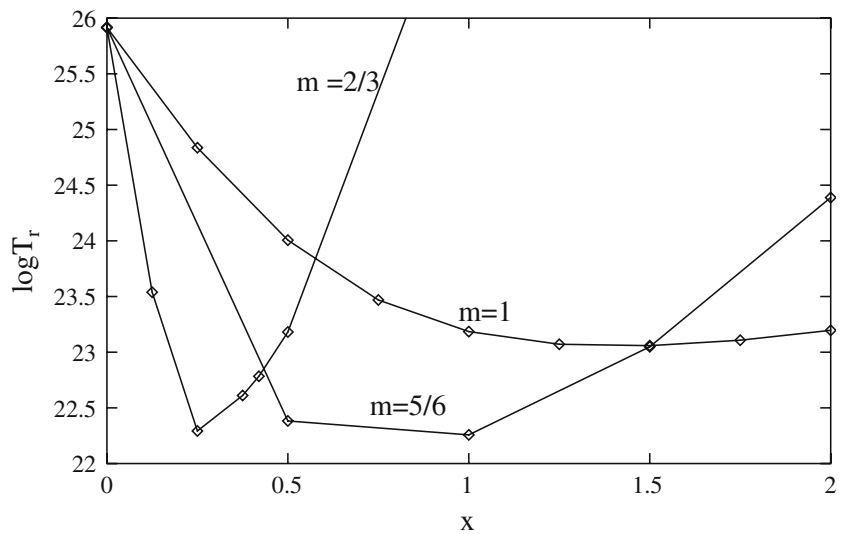

Fig. 11. Plot of $\log T_{n}$ for $m=2 / 3,5 / 6$ and 1 as a function of $x$ for $n=1000$.

the behaviour of various prefactors, we study this numerically. Figure 11 shows plot of $T_{r}$ for $r=1000$, for $m=2 / 3,5 / 6$ and 1 as a function of $x$. For $1 \geqslant a \geqslant 2 / 3$, to leading order $T_{r}$ goes as $\exp \left(c r^{1 / 3}\right)$, but there exist a nontrivial value of $x$ at which $T_{r}$ is minimum for a given $m$. If we look at $T_{r}$ at best value of $x$ for $m=2 / 3,5 / 6$ and 1 , we find that as $r$ increases the difference is not significant.

Hence we conclude that to leading order, $T_{r} \sim \exp \left(c r^{1 / 3}\right)$, for the best choice of $p$. For all $2 / 3 \leqslant m \leqslant 1$, there exist a range of $x$ for which the time complexity of the algorithm will remain qualitatively the same.

\subsection{Lattice Animals on a 2-Dimensional Square Lattice}

We also studied the efficiency for LA on a square lattice. From exact series enumeration the $A_{r}$ is known to vary as $\lambda^{r}$ with $\lambda \approx 4.06257 .{ }^{(26)}$ In this case also the number of offsprings a node at level $r$ can have is $O(r)$ and the genealogical tree in this case though more complicated, is qualitatively similar. Numerically, we find that the probability distribution of number of descendants $k$ (of a randomly chosen node) has a maximum at $k=4$, with $\operatorname{Prob}(k=4) \approx 1 / 4$. We enumerated lattice animals up-to sizes 1000 using IE with $10^{6} \mathrm{MC}$ runs. It took time of order one day on a Pentium-4 machine. With IIE we generated samples of size 2000 with $2 \times 10^{6} \mathrm{MC}$ runs in 2-3 days time. These sizes are of same order as those produced using the cut and paste type algorithms.

In this case, we find that $P(r)$ has the stretched exponential form $P(n) \sim \exp \left(-c n^{\alpha}\right)$, with $\alpha \approx 0.4$ for both IE and IIE. Figure 12 shows 


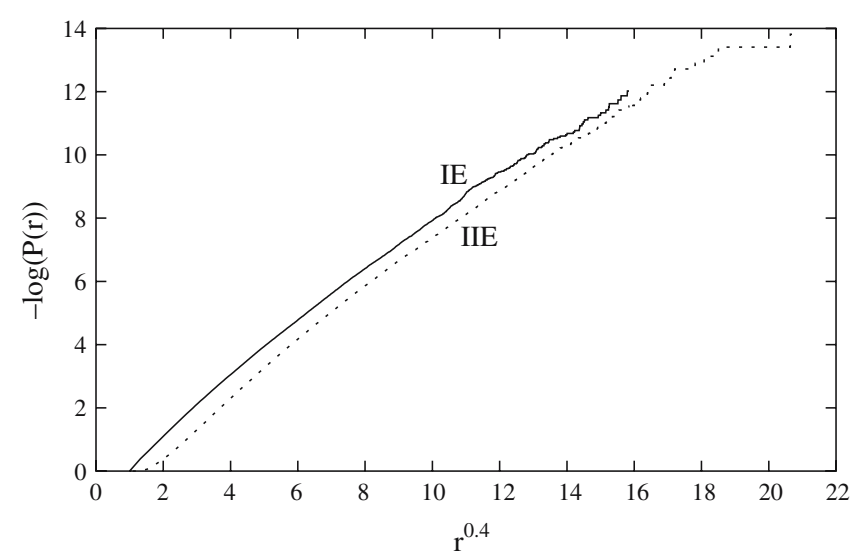

Fig. 12. Plot of $-\log (P(2, r))$ versus $r^{0.4}$ for lattice animals on a square lattice with IE and IIE.

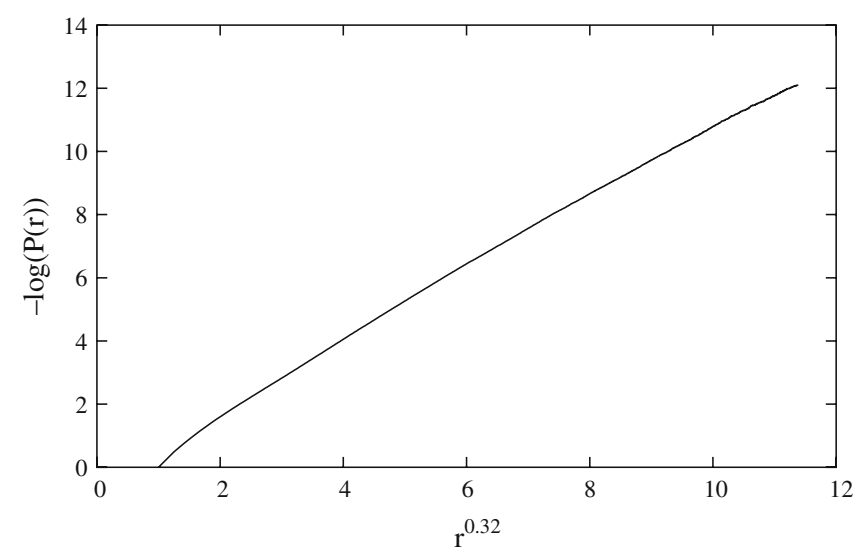

Fig. 13. Plot of $-\log (P(r))$ versus $r^{0.32}$ for directed animals with IIE.

$[-\log P(r)]$ varies approximately linearly with $r^{0.4}$. We also studied the directed LA on a square lattice. In this case we find that, $\alpha=0.32 \pm 0.02$ (Fig. 13).

\section{DISCUSSION}

We find the efficiency of IE to be different for linear and branched polymers. This is due to the fact that genealogical tree for the latter is much more nonuniform. 
For SAWs, in any dimension, the time to generate an independent sample of $n$ steps $T_{n} \sim a_{d} n^{2}$, independent of dimension for both IE and IIE. For IE there is no significant change in $a_{d}$ with dimension. But for IIE $a_{d} \sim d^{-2}$. In the limiting case of SAW on binary tree $T_{n}=n$ for IIE.

For branched polymers $T_{n}$ increases as $\exp \left(c n^{\alpha}\right)$ with $0<\alpha<1$ in all dimensions for both IE and IIE. Redistributing weight does not change the value of $\alpha$. The IIE works better than IE, but the difference is only in the coefficient $c$. The exponent $\alpha$ depends weakly on the dimension, its relation to the usually studied exponents of the branched polymer problem, e.g. $\theta$, $v$ is not clear at present.

As discussed earlier, the genealogical tree for cluster enumeration is not unique and one might argue that Martin's scheme is not the optimal choice. We tried to generate the genealogical tree using some variations of this rule, but we did not find any significant change in efficiency of the algorithm.

For branched polymers, the degree of a node in the genealogical tree is not bounded, and the maximum degree increases with depth of the genealogical tree. However, the fractional number of nodes with high degree is very small. For genealogical tree corresponding to animals on a binary tree we find the fractional number of $k$-nodes goes down exponentially with $k$ for large $k$ (Eq. (18)). Similar, behaviour was observed for branched polymers and directed branched polymers on a square lattice numerically. It is surprising that even an exponentially rare distribution of nodes with large degree seems to be enough to change the behaviour of efficiency of the algorithm on the tree.

In the case of branched polymers, we found that the $T_{n}$ for IE varies as $\exp \left(c n^{\alpha}\right)$ with $0<\alpha<1$. While this is not very good, one can find problems for which IE's performance is even worse with $\alpha=1$. As an example, consider SAWs on a disordered lattice, obtained by removing a fraction $(1-u)$ of bonds at random from a square lattice. It is known that the average number of SAWs of length $n$ varies as $(u \lambda)^{n}$, (27) where $\lambda$ is the growth constant of the SAWs on the same lattice with $u=1$. Hence the growth constant of the corresponding genealogical tree would also be $u \lambda$. Now if we consider a square lattice, the $\lambda \approx 2.638$ and the bond percolation threshold is $1 / 2$. For $1 / \lambda<u<1 / 2$, all clusters would be finite with probability 1 , and the probability that cluster contains $n$ sites would decrease exponentially with $n$. In this case, IE will be inefficient and even for best choice, $T_{n}$ will vary as $\exp (c n)$.

One could argue that IE is a rather inefficient algorithm, which gives reasonable performance only for a small selected set of problems. We do not think so. In fact, the causes that make IE inefficient are also operative in the much larger class of genetic type algorithms. The high degree of correlations between different samples generated is a common feature of many of these algorithms which employ pruning and enrichment. For 
example, one could expect a similar behaviour to occur in the Berreti-Sokal algorithm, ${ }^{(10)}$ for branched polymers. The correlations arise because in all such 'evolutionary' type algorithms different samples generated often share a common ancestor in the past. Whether our results can be generalised to a larger class of PERM type algorithms seems to be an interesting question for further study.

\section{APPENDIX A}

As discussed in Section 1, to enumerate all allowed configurations on a computer, one need a good exact enumeration algorithm which would generate all possible configurations exactly once, without needing to refer to what has been generated previously. Hence, one has to label the $n$-point configurations such that for any $n$-point configuration the labelling is unique and on removing the last added site we must get an allowed $(n-1)$-point configuration. For the self avoiding walks this can be easily achieved by labelling the first point of walk as one, the second two, and so on. But usually such natural choice of labelling doesn't exist for most problems. For lattice trees and animals, Martin discusses this in detail. ${ }^{(17)}$ Here we describe briefly his algorithm for labelling a $n$-cluster.

- Choose a rule for ordering the neighbours of any given site. For example, for LA on a square lattice (Fig.1), we chose the rule that the upward neighbour is labelled before the right neighbour. For LA on the binary tree we choose left neighbour before the right neighbour. (Fig. 6).

- We label the root as one and its neighbours are labelled 2,3,4 . in the order according to the priority rule.

- When all points adjacent to point 1 have been labelled, label any still unlabelled points adjacent to point 2 according to the priority rule and then of point 3 and so on. This labeling hence induces a tree structure on the cluster which is the genealogical tree.

The labelling described above is just one way of labelling the configurations. One can invent many other labelling schemes, which would give rise to different genealogical tree. But we find that the qualitative nature genealogical tree does not depend on the rules of labelling.

\section{ACKNOWLEDGMENTS}

We thank M.Barma for a careful reading of the manuscript, and the referee for many helpful remarks, which have helped improve our presentation. 


\section{REFERENCES}

1. A. D. Sokal, Monte-Carlo Methods for the Self Avoiding Walk 1995, in Monte Carlo and Molecular Dynamics Simulations in Polymer Science, K. Binder, ed. Oxford University Press New York 47-124, hep-lat/9405016.

2. M. Doi and S. F. Edwards, The Theroy of Polymer Dynamics (Clarendon Press, Oxford, 1986).

3. F. T. Wall and F. Mandel, Macromolecular dimensions obtained by an efficient Monte Carlo method without sample attrition, J. Chem. Phys. 63:4592-4595 (1975).

4. N. Madras and A. D. Sokal, The pivot algorithm: A highly efficient Monte Carlo Method for the self-avoiding walk, J. Stat. Phys. 50:109 (1988).

5. S. Caracciolo, A. Pelissetto, and A. D. Sokal, A nonlocal Monte Carlo algorithm for self-avoiding walks with fixed endpoints, J. Stat. Phys. 60:1 (1990).

6. F. T. Wall and J. J. Erpenbeck, New method for the statistical computation of polymer dimensions, J. Chem. Phys. 30:634-637 (1959).

7. P. Grassberger and W. Nadler, "Go with the winners - Simulations, condmat/0010265", Proc. der Heraeus-Ferienschule "Vom Billiardtisch bis Monte Carlo: Spielfelder der statistischen Physik", (Chemnitz, October 2000).

8. E. J. Janse van Rensburg, S. G. Whittington, and N. Madras, The pivot algorithm and polygons: Results on the FCC lattice, J. Phys. A 23:1589 (1990).

9. T. Kennedy, A faster implementation of the pivot algorithm for self-avoiding walks, J. Stat. Phys. 106:407-429 (2002).

10. A. Berreti and A. D. Sokal, New Monte Carlo method for self-avoiding walk, J. Stat. Phys. 40:483 (1985).

11. E. J. Janse van Rensburg, and N. Madras, A nonlocal Monte Carlo algorithm for lattice trees, J. Phys. A:Math. Gen. 25:303-333 (1992); Metropolis Monte Carlo simulation of lattice animals, J. Phys. A:Math. Gen. 30:8035-8066 (1997); E. J. Janse van Rensburg and A. Rechnitzer, High precision canonical Monte Carlo determination of the growth constant of square lattice trees, Phys. Rev E 67:0361161-0361169 (2003).

12. S. You and E. J. Janse van Rensburg, Adsorbing trees in two dimensions: A Monte Carlo study, Phys. Rev. E 64:0461011-0461019 (2001).

13. H. P. Hsu, W. Nadler, and P. Grassberger, Simulations of lattice animals and trees, cond-mat/0408061 (2004).

14. S. Redner and P. J. Reynolds, Position-space renormalisation group for isolated polymer chains, J. Phys. A 14:2679 (1981).

15. D. Dhar and P. M. Lam, A Monte Carlo method for series expansions, J. Phys. A: Math. Gen. 19:L1057-1061 (1986).

16. P. M. Lam, Monte Carlo study of lattice animals in $d$ dimensions, Phys. Rev. A 34:2339-2345 (1986).

17. J. L. Martin, Computer Techniques for Evaluating Lattice Constants, Phase Transitions and Critical Phenomena, C. Domb and O. M. Green, eds. (vol. 3, Academic Press London, 1983).

18. T. E. Harris, Theory of Branching Processes, (Springer-Verlag, Berlin, 1963).

19. G. Grimmett, Percolation (Springer-Verlag, Berlin, 1989).

20. N. Madras and G. Slade, The Self Avoiding Walk (Birkhauser Boston, 1993).

21. B. Nienhuis, Exact critical point and critical exponents of $\mathrm{O}(\mathrm{n})$ models in two dimensions, Phys. Rev. Letts. 49:1062 (1982).

22. I. Jensen and A. J. Guttmann, Self-avoiding polygons on the square lattice, condmat/9905291, (1999). 
23. R. P. Stanley, Enumerative Combinatorics, (Vol.2, Chapter 6, Cambridge University Press, Cambridge, New York, 1999).

24. W. V. Saarloos, Front propagation into unstable states, Phys. Rep. 386:29 (2003).

25. E. Brunet and B. Derrida, Shift in the velocity of a front due to a cutoff, Phys. Rev. E 56:2597 (1997); S. N. Majumdar and P. L. Kaprivsky, Extreme value statistics and travelling fronts: Various applications, Physica A 318:161 (2003).

26. I. Jensen, Enumerations of lattice animals and trees, J. Stat. Phys. 102:865-881 (2001).

27. K. Barat and B. K. Chakrabarti, Statistics of self-avoiding walks on random lattices, Phys. Rep. 258:377 (1995). 